\title{
Experimental Characterization of the Jet Wiping Process
}

\author{
Miguel Alfonso Mendez ${ }^{1, \star}$, Adriana Enache ${ }^{1}$, Anne Gosset ${ }^{2,}$, and Jean-Marie Buchlin ${ }^{1}$ \\ ${ }^{1}$ EA Dep., von Karman Institute for Fluid Dynamics, Sint-Genesius-Rode, Belgium \\ ${ }^{2} \mathrm{Naval}$ and Oceanic Engineering Department, University of A Coruña, Ferrol, Spain
}

\begin{abstract}
This paper presents an experimental characterization of the jet wiping process, used in continuous coating applications to control the thickness of a liquid coat using an impinging gas jet. Time Resolved Particle Image Velocimetry (TR-PIV) is used to characterize the impinging gas flow, while an automatic interface detection algorithm is developed to track the liquid interface at the impact. The study of the flow interaction is combined with time resolved 3D thickness measurements of the liquid film remaining after the wiping, via Time Resolved Light Absorption (TR-LAbs). The simultaneous frequency analysis of liquid and gas flows allows to correlate their respective instability, provide an experimental data set for the validation of numerical studies and allows for formulating a working hypothesis on the origin of the coat non-uniformity encountered in many jet wiping processes.
\end{abstract}

\section{Introduction}

In the jet wiping process, an impinging gas jet is used to control the thickness of a liquid coating dragged by a moving substrate. The substrate is typically a large sheet withdrawn vertically from a bath at a constant speed so that the removed liquid runs back by gravity. This process is widely used in photographic film manufacturing, paper industry and in the hot-dip galvanizing process [1-4].

Because of its industrial relevance, the development of jet wiping lines has been supported by a large number of analytical and experimental investigations, which led to the formulation of several simplified physical models [59]. These models provide the engineering tools to predict the final coating thickness as a function of all the operating parameters and therefore provide the framework for the automatic control of these lines [10-12].

The drawback of the method, on the other hand, is the limited surface quality achievable, which constitute a major concern for applications demanding high surface finish quality such as, for example, automotive industry. Understanding the mechanisms responsible for the coating nonuniformities requires a time-resolved characterization of the interaction between the gas jet flow and the liquid film, up to day only achieved by means of numerical simulations [13-15] or simplified laboratory models of the wiping process [16-18]. Most of the experimental works on the jet wiping, in fact, have been focused on average quantities to validate physical models $[8,19,20]$ or in the definition of operational maps to avoid the liquid film break up, a phenomenon known as splashing [20, 21].

This work provides a time resolved experimental characterization of the jet wiping process combining Time Re-

\footnotetext{
^e-mail: mendez@vki.ac.be
}

solved Particle Image Velocimetry (TR-PIV) on the gas jet with an automatic tracking of the liquid interface and Time Resolved Light Absorption (LAbs) thickness measurements of the final coat.

The parameters and the dimensionless quantities governing the process are described in Section 2, recalling the operative range of the method in several industrial applications. The experiments were conducted in the Ondule laboratory, developed at the von Karman Institute (VKI) to simulate the jet wiping process. The facility and its instrumentation are presented in Section 3.

The data processing of each measurement technique is presented in Section 4. These include the image preprocessing by means of an automatic masking algorithm to track the gas liquid interface from the TR-PIV images (Section 4.1, [18, 22-24]), the gas flow field modal analysis via Proper Orthogonal Decomposition (POD, Section $4.2,[25,26])$ and the 3D film thickness reconstruction of the final coat (Section 4.3, [23, 27-29]). Each of these techniques is used to construct a characteristic frequency spectrum for the gas jet flow, the final coat and the liquid layer removed in the wiping process, referred to as runback flow. These spectra are presented and discussed in Section 5; the conclusions are drawn in Section 6.

\section{Problem Formulation and Scaling Laws}

A schematic of the jet wiping process in its basic configuration is proposed in Figure 1, recalling the process parameters: the substrate speed $U_{s}$, the nozzle stagnation pressure $\Delta P_{n}$, the nozzle opening $d$ and the nozzle stand-off distance $Z$. The relevant liquid properties are density $\rho_{l}$, dynamic viscosity $\mu_{l}$ and surface tension $\sigma_{l}$.

This section aims at presenting the dimensionless numbers and the reference quantities used to scale the 
measurements presented in this work and to discuss the similarity between the investigated configuration and the ones encountered in different industrial processes. These scaling parameters are inferred from the formulation of the simplified models used in the literature of the jet wiping process.

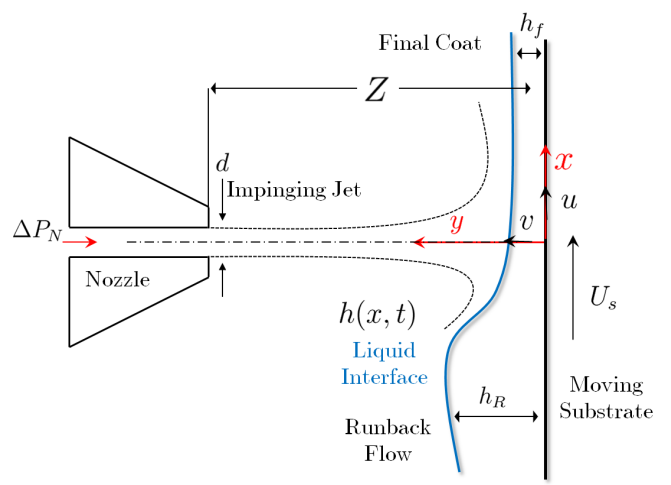

Fig. 1. Schematic of the jet wiping process: a nozzle at a pressure $\Delta P_{N}$ releases a jet flow at a distance $Z$ from a substrate moving at a speed $U_{s}$. The impingement produces a run-back liquid layer of thickness $h_{R}$ and a thinner final coat of thickness $h_{f}$.

Simplified models build from the governing equations of the thin liquid film flow, considered an incompressible flow with constant properties, under the assumption of lubrication theory $\left(\partial_{x} h \rightarrow 0, v \rightarrow 0, \partial_{x} \rightarrow 0\right)$ and steady state $\left(\partial_{t} \rightarrow 0\right)$ conditions. It is thus assumed that the liquid film has a boundary layer behavior $\left(\partial_{y} p \rightarrow 0\right)$ and that the coupling between the two phases is one-way: the pressure and the shear stress distribution produced at the wall by the impinging gas jet are not influenced by the liquid film, and are therefore inferred from well established experimental correlations obtained for jet impinging on a dry wall. Under these conditions, and neglecting the effects of surface tension, the film thickness at each stream-wise coordinate must satisfy the following dimensionless cubic equation [6-9]:

$$
\left(\partial_{\hat{x}} \hat{P}_{g}+1\right) \hat{h}^{3}-\frac{3}{2} \hat{T}_{g} \hat{h}^{2}-\hat{h}+2 \hat{q}=0
$$

where $\hat{h}=h / h_{0}$ is the dimensionless film thickness, with $h_{0}=\sqrt{v U_{s} / g}$ the reference thickness; $\partial_{\hat{x}} \hat{P}_{g}=$ $\partial_{x} \hat{P}_{g} /\left(\rho_{l} g\right)$ and $\hat{T}_{g}=T_{g} / \sqrt{\mu_{l} \rho_{l} U_{s} g}$ are the dimensionless pressure gradient and shear stress distributions produced by the impinging gas jet; $\hat{q}=2 q / 3 \sqrt{v U_{s}^{3} / g}$ is the net dimensionless flow rate remaining in the final coat. Simplified physical models differ in the degree of simplification involved in the solution of eq.(1), which can include the surface tension $[8,30]$, neglect the shear stress contribution [5], or force the simplification by assuming that the maximum pressure gradient $\left(\partial_{\hat{x}} \hat{\mathcal{P}}_{g}\right)$ and the maximum shear stress $\left(\hat{\mathcal{T}}_{g}\right)$ act in the same location [8]. In this case, assuming that the system operates in optimal conditions $\left(\partial_{h} q=0\right)$, the final thickness $\hat{h}_{f}$ obeys a simple equation:

$$
\hat{h}_{f}=\frac{\hat{\mathcal{T}}_{g}+\sqrt{\hat{\mathcal{T}}_{g}^{2}+4\left(\partial_{\hat{x}} \hat{\mathcal{P}}_{g}+1\right)}}{2\left(\partial_{\hat{x}} \hat{\mathcal{P}}_{g}+1\right)} .
$$

The maximum dimensionless pressure gradient, from the correlations in $[31,32]$, reads

$$
\partial_{x} \mathcal{P}_{g}=\left(\frac{0.7141}{b} P_{M}\right) \frac{1}{\rho_{l} g},
$$

where

$$
\begin{aligned}
P_{M} & =6.5 \Delta P_{n} \frac{d}{Z} \\
b & =\left[0.0019\left(\frac{Z}{d}\right)^{2}+0.0551\left(\frac{Z}{d}\right)+0.4035\right] d .
\end{aligned}
$$

Similarly, the maximum dimensionless shear stress is:

$$
\hat{\mathcal{T}}_{g}=-\left(P_{n} C_{\tau} \frac{d}{Z}\right) \frac{1}{\sqrt{\mu_{l} \rho_{l} U_{s} g}},
$$

where the constant $C_{\tau}$ is a function of the Reynolds number $R e_{J}=U_{J} d / v_{g}$ of the gas jet:

$$
C_{\tau}= \begin{cases}0.1287-10^{-5} R e_{j} & \text { if } R e_{J} \leq 6170 \\ 0.067 & \text { if } R e_{J}>6170\end{cases}
$$

The validity of this approximated model for predicting the final thickness is limited to the case of strong wiping $\left(\partial_{x} \hat{P}_{g} \gg 1\right)$, as discussed in [8] and [30]. In the regimes of weak wiping $\left(\partial_{x} \hat{P}_{g} \sim 1\right)$, where surface tension becomes important, this model is known to significantly over-predict the wiping capabilities of the jet flow. Moreover, the extreme simplifications involved in the derivation of eq.(2) do not allow for investigating the jet wiping process in the dynamic conditions, i.e. including both splashing occurrence [20] and film instability [36]. Yet, this physical model is indicative of the impact of the jet parameters on the liquid flow. In this work it is used as a benchmark and to derive the scaling quantities for the non-dimensionalization of the measurements. These dimensionless numbers, moreover, allows for investigating the flow similarity between the laboratory conditions and the conditions of several industrial processes.

It is interesting to observe, for example, that under the assumption leading to eq.(2), the thickness of the run-back flow $\hat{h}_{R}$ is linked to the final thickness $\hat{h}_{f}$ by mass conservation. In the far field conditions $(x \rightarrow \pm \infty)$, in fact, where $P_{g} \rightarrow 0$ and $T_{g} \rightarrow 0$, eq.(1) reduces to a universal cubic equation, shown in Fig. 2 for an exemplary case. This cubic equation results from the balance of viscous and gravitational forces and has two admissible solutions for a given flow rate $\hat{q}$. Fixing the first, corresponding to the final thickness $\hat{h}_{f}$ in eq.(2), the second is easily computed numerically. This thicker solution corresponds to the run-back flow thickness $\hat{h}_{R}$.

In absence of jet wiping, then, as only one solution is possible, the coating thickness withdrawn by the plate 
Table 1. Indicative operation range of the jet wiping processes in several industrial configuration, including the VKI jet wiping laboratory Ondule used in this work. The references from which the data are taken (or inferred from) are indicated in each entry. The final coating thickness and the run-back flow thickness obtained using the 0D wiping model described in Section 2.

\begin{tabular}{ccccccccc} 
& \multicolumn{7}{c}{ Dimensional Quantities (Indicative values) } \\
\cline { 2 - 9 } & $\begin{array}{c}\rho_{l} \\
{\left[\mathrm{~g} / \mathrm{cm}^{3}\right]}\end{array}$ & $\begin{array}{c}\mu \\
{[\mathrm{cP}]}\end{array}$ & $\begin{array}{c}U_{s} \\
{[\mathrm{~m} / \mathrm{min}]}\end{array}$ & $\begin{array}{c}U_{J} \\
{[\mathrm{~m} / \mathrm{s}]}\end{array}$ & $\begin{array}{c}d \\
{[\mathrm{~mm}]}\end{array}$ & $\begin{array}{l}Z \\
{[\mathrm{~mm}]}\end{array}$ & $\begin{array}{c}h_{f} \\
{[\mathrm{~mm}]}\end{array}$ & $\begin{array}{c}h_{R} \\
{[\mathrm{~mm}]}\end{array}$ \\
\hline Ondule Laboratory & 1.02 & $50-100$ & $6-30$ & $20-40$ & 1.3 & $13-20$ & $0.2-0.5$ & $1.5-2.5$ \\
Photographic Film Coater [1,3,33] & $1-1.1$ & $1-40$ & $10-100$ & $15-30$ & $0.5-1$ & $2-10$ & $0.05-0.2$ & $0.4-3$ \\
Paper Coater [2, 34, 35] & $0.8-1.5$ & $5-300$ & $400-700$ & $50-150$ & $3-10$ & $20-70$ & $0.2-1$ & $4-15$ \\
Galvanizing Line [4-6] & 6.54 & $2.5-3.5$ & $50-150$ & $100-250$ & $1-1.5$ & $6-20$ & $0.01-0.07$ & $0.3-0.5$ \\
\hline
\end{tabular}

Table 2. Reference dimensionless quantities for the ranges presented in Table.1. The dimensionless pressure gradient and shear stress are computed using the correlations in (3) and (5). The final coating thickness $\hat{h}_{f}$ and the run-back $\hat{h}_{R}$ are scaled with respect to the optimal thickness $h_{0}=\sqrt{v_{l} U_{s} / g}$ and the film thickness ratio is defined in eq.(7). The Reynolds number in the jet flow and the liquid film are defined as $R e_{J}=U_{J} d / v_{g}$ and $R e_{L}=U_{s} h_{0} / v_{l}$.

\begin{tabular}{cccccccc}
\cline { 2 - 8 } & \multicolumn{7}{c}{ Dimensionless Quantities } \\
\cline { 2 - 8 } & $\partial_{x} \mathcal{P}^{*}$ & $\mathcal{T}^{*}$ & $\hat{h}_{f}$ & $\hat{h}_{R}$ & $\zeta$ & $\operatorname{Re}_{l}\left(\times 10^{3}\right)$ & $\operatorname{Re}_{J}\left(\times 10^{3}\right)$ \\
\hline Ondule Laboratory & $10-30$ & $0.4-1$ & $0.2-0.3$ & $1.5-1.6$ & $1.2-1.4$ & $0.001-0.01$ & $2.5-3.5$ \\
Photographic Film Coater [1, 3, 33] & $35-40$ & $0.2-3$ & $0.15-0.2$ & $1.65-1.7$ & $1.4-1.5$ & $0.01-0.04$ & $1-1.5$ \\
Paper Coater [2, 34, 35] & $6-400$ & $0.2-2.5$ & $0.05-0.4$ & $1.5-1.7$ & $1.1-1.6$ & $0.4-5$ & $10-300$ \\
Galvanizing Line [4-6] & $30-600$ & $2-40$ & $0.08-0.23$ & $1.6-1.65$ & $1.3-1.6$ & $0.3-0.9$ & $10-25$ \\
\hline
\end{tabular}

is ${ }^{1}$ the one corresponding to the maximum flux. Since this point is considered to build the reference quantities, in such conditions one recovers $\hat{q}=1$ and $\hat{h}=1$.

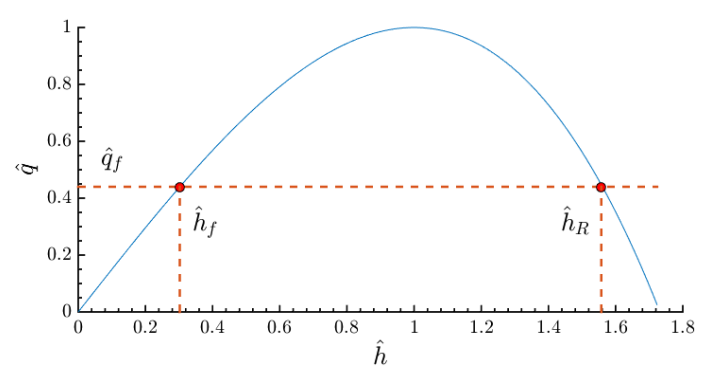

Fig. 2. Cubic relation between coating thickness $\hat{h}$ and flow rate $\hat{q}$ in the far field conditions for eq.(1). For a given flow rate, two possible solutions are possible. The first corresponds to the final thickness $\hat{h}_{f}$ (estimated from eq.2); the second corresponds to the run-back flow thickness $\hat{h}_{R}$.

The relevant dimensional and dimensionless parameters for the experimental set up used in this work are collected in Tables 1 and 2, together with those encountered in typical industrial configurations such as photographic film manufacturing [1, 3, 33], paper industry [2, 34, 35] and galvanizing lines [4-6]. In addition to the aforementioned dimensionless quantities, the table includes the

\footnotetext{
${ }^{1}$ This results, independent of surface tension, is valid for sufficiently high Reynolds and Capillary numbers. For a discussion on the free-drag out problem the reader is referred to [6, 37-39].
}

Reynolds number in the liquid film $R e_{L}=U_{s} h_{0} / v_{l}$ and the wiping thickness aspect ratio $\zeta$, defined as:

$$
\zeta=\frac{h_{R}-h_{f}}{h_{0}}
$$

Although only indicative, these ranges give an overview of the variety of operating conditions in which the jet wiping process is used: from the extremely high jet velocity used in the galvanizing lines, to the large viscosities encountered in a paper coater and the thinnest coatings used in photographic film manufacturing. Yet, there exists a wide range of dimensionless film thickness which overlaps among all the configurations and leads to similar average thickness profiles. The Ondule facility, presented in Section 3, was designed at the von Karman Institute (VKI) to cover this range while operating in conditions for which the nonintrusive experimental techniques presented in Section 3 and Section 4 give adequate accuracy and resolution.

\section{The VKI Ondule Facility}

The jet wiping facility Ondule is described in Fig.3a. In this simplified laboratory model, the rising substrate is simulated by a cylinder (1) in acrylic glass with $450 \mathrm{~mm}$ diameter and $350 \mathrm{~mm}$ width. This cylinder is dipped in a liquid bath (2) for about $5 \mathrm{~cm}$ and it is driven by an electrical motor rotating at speed in the range $5-20 \mathrm{rpm}$ $\left(U_{s} \approx 0.1-0.45 \mathrm{~m} / \mathrm{s}\right)$. A doctoring blade (3) removes the coating layer before immersion. 


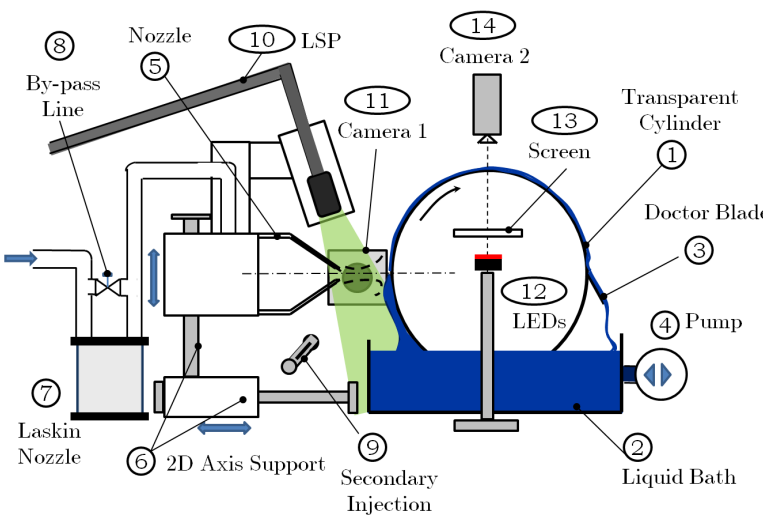

a)

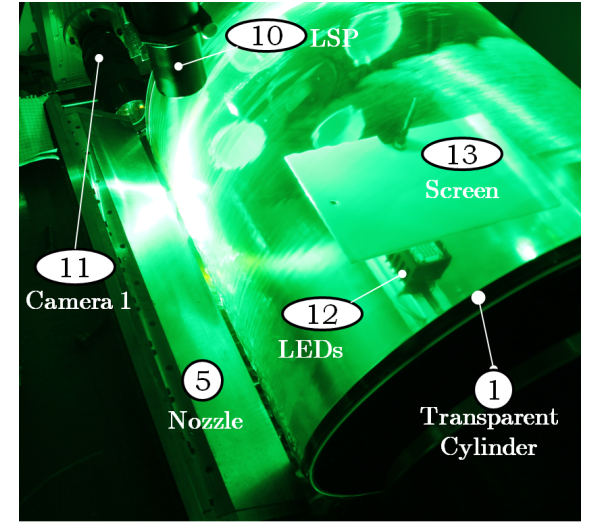

b)

Fig. 3. a) sketch of the VKI Ondule facility used to reproduce the jet wiping process in Fig.1, along with the required hardware for the TR-PIV and TR-LAbs measurements. Figure b: photograph of the test section showing some of the key components listed in Figure a: the nozzle (5), the PIV camera (11) and the optical arm (10), the transparent cylinder (1) hosting the LEDs (12) and the screen (13) for the liquid film back-lightning.

The working liquid is Dipropylene Glycol $\left(\rho_{l}=1026 \pm\right.$ $\left.1 \% \mathrm{Kg} / \mathrm{m}^{3}, \mu=50 \pm 2 \% \mathrm{cP}, \sigma=32 \pm 1 \% \mathrm{~N} / \mathrm{mm}\right)$, introduced in the bath by a volumetric pump Pollard PR40 (4) at the beginning of the test. The nozzle (5) releasing the gas jet is installed on a two axis mechanism (6) to adjust the impingement position and the stand-off distance.

To perform TR-PIV and flow visualization, the nozzle is supplied by a Laskin Nozzle PIVTEC45-M (7 in Fig.3a) operating with mineral oil Ondina Shell 917 to provide seeding particles. An additional bypass line (8) allows to control the seeding density in the jet flow and to supply in parallel a secondary injection (9) positioned below the jet flow to maximize the seeding uniformity. The light source is a dual diode-pumped ND:YLF laser (Quantronix Darwin Duo 527), equipped with a light sheet probe LSO from ILA (10) to produce the laser sheet. The interaction between the jet flow and the liquid film is recorded by a CMOS Photron FASTCAM SA1 (11) positioned on one side of the facility at a distance of about $16 \mathrm{~cm}$ from the investigated plane. This camera is equipped with a zoom objective Tamron CZ-735 70 - 300mm, providing a scaling factor of $20 \pm 0.1 \mathrm{pixel} / \mathrm{mm}$. The video size is $5.5 \mathrm{~GB}$, corresponding to $n_{t}=21000$ images (10500 image pairs) with a resolution of $512 \times 512$ pixels, taken at a frequency of $f_{s}=3 \mathrm{kHz}$. In order to enhance the interface visibility via laser induced fluorescence, Rhodamine B is diluted in the liquid with a concentration of $\approx 10 \mathrm{mg} / \mathrm{Kg}$. An exemplary image of the TR-PIV acquisition is shown in Fig.4.

To perform TR-LAbs, a diffuse light source is installed inside the transparent cylinder. This consists of two arrays of $4 \times 4$ LEDs of $\approx 0.7 \mathrm{~W}$ each, with emittance in the range of $636 \pm 26 \mu m$ (12 in Fig.3a). These LEDs are installed behind a $3 \mathrm{~mm}$ thick sheet of Opaline acrylic glass (13). The receiver (14) is a rolling shutter 16 bits CMOS camera (Hamamatsu ORCE-Flash 4.0) synchronized with the LEDs, to acquire 2000 back-lighting images at $300 \mathrm{~Hz}$ with a resolution of $660 \times 1800$ pixels. This camera is installed perpendicularly to the test section, at about $40 \mathrm{~cm}$ from the cylinder. It is equipped with a $35 \mathrm{~mm}$ Nikkor-s objective, yielding a scaling factor of $13.4 \pm 0.3 \mathrm{pixel} / \mathrm{mm}$, and a band-pass filter (band pass range $640 \pm 40 \mu \mathrm{m}$ ) to limit the camera sensitivity in the wavelength range where the light absorption takes places. To maximize and match the absorption spectra of the liquid with the emission spectra of the light, a methylene blue colorant is diluted in the liquid, with a concentration of $21 \mathrm{mg} / \mathrm{Kg}$. The definition of the optimal colorant concentration for minimizing the measurement uncertainties is presented in [29, 40]. An exemplary image of the TR-Labs acquisition is shown in Fig.5 (top), together with the corresponding reference image (bottom) of the dry test section.

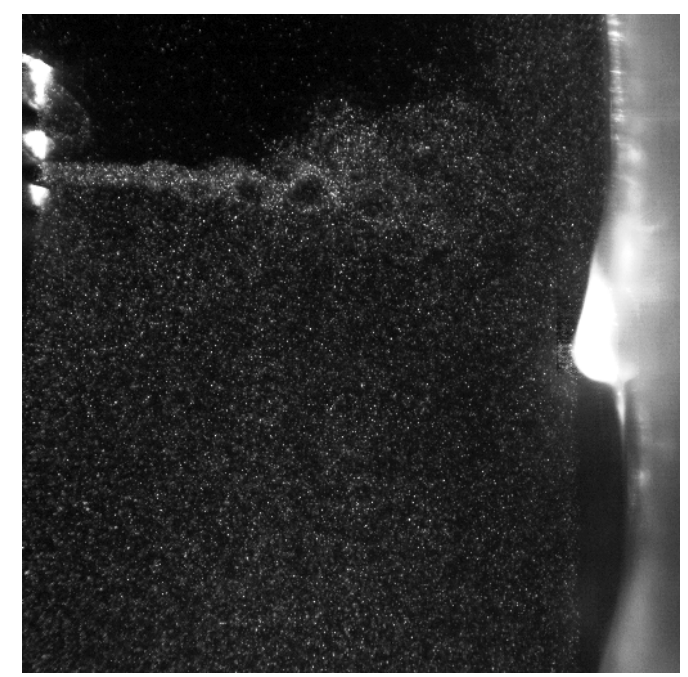

Fig. 4. Exemplary TR-PIV image of the gas jet liquid film interaction. The liquid film visibility is enhanced by the laser induced fluorescence. Both seeding injections $(4,5$ in Fig.3 a) are active, producing a uniform seeding.

A picture of the test section with some of the key components for both measurement techniques is shown in Fig.3b. 

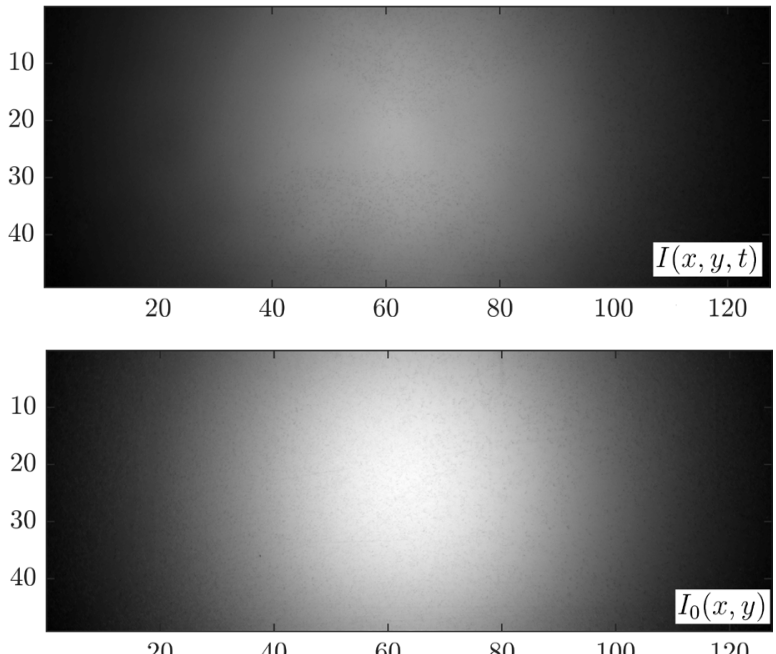

20

60

100

Fig. 5. Exemplary TR-LAbs image of the final coat (top) and corresponding reference image (bottom).

\section{Data Processing}

\subsection{Runback Flow Analysis and Masking}

The liquid interface is monitored from the PIV images using an image processing routine developed in Matlab. This routine consists of four steps. First, the PIV images are cropped around the liquid interface, as shown in Fig.6, for the example image presented in Fig.4. Second, the set of images is filtered by means of the POD-based masking procedure described in $[23,24]$. This filter consists of decomposing the video sequence via Proper Orthogonal Decomposition (POD) and keeping only a small fraction of the dominant modes, in order to capture the motion of large objects while removing the uncorrelated contribution of the particle pattern. The result of this step is the complete removal of the PIV particles from the images, as shown in Fig.6b. This operation significantly simplifies the interface detection by means of standard edge enhancement methods, performed by using a Gaussian sharpening kernel (Fig.6-c) and followed by the peak detection of the gray scale profiles along the image horizontal lines. The result is finally filtered by means of a Savitzky-Golay smoothing filter [41]. For the exemplary image sample proposed, the interface is shown in Fig.6-d.

The interface detection in each time step is used to assembly the characteristic map $h(x, t)$, describing the propagation of interface disturbances in space and time. An example of such map is shown in Fig.7a. This map displays the characteristic lines along which the waves in the impinged liquid film propagates. The automatic detection of these lines and their slope, related to the propagating wave speed, is carried out by means of Hough Transform as described in [29]. The characteristic spectrum of the run back liquid film is constructed by choosing a location in space $x^{*}$ (indicated in Fig.7a) and computing its spectra $\mathcal{H}_{r}(f)$ using the Welch's spectral density estimation $[42,43]$ with 5 windows and $30 \%$ overlap. A typical result is shown in Fig.7b.

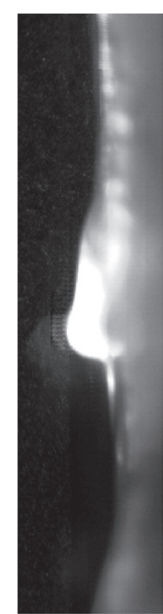

a)

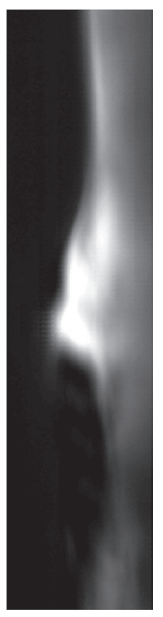

b)

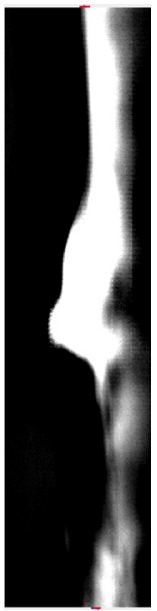

c)

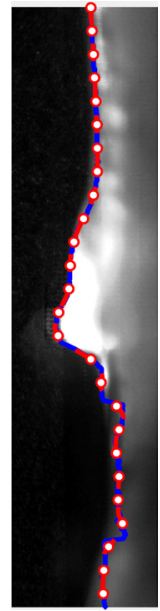

d)
Fig. 6. Image processing steps for the automatic liquid interface tracking. Each image is cropped in the portion displaying the liquid (a), and filtered using a POD background removal algorithm (b) to remove the PIV particles. The result is sharpened via Gaussian Kernel (c) and the interface retrieved by gray scale gradient analysis (d).

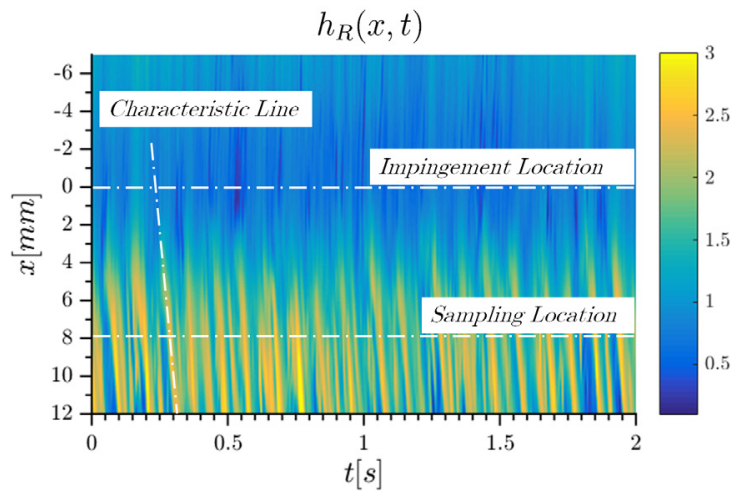

a)

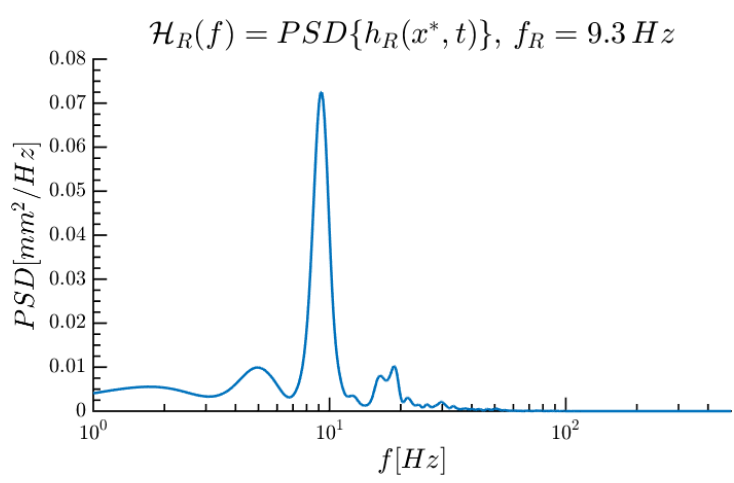

b)

Fig. 7. Exemplary characteristic map of the liquid interface retrieved from the TR-PIV images. This map shows the characteristic lines of the wave propagation and it is used to compute the characteristic spectrum of the run back liquid film, shown in b).

\subsection{Jet Flow Analysis via Modal Decomposition}

The PIV images are first masked using the interface detection described in the previous section and then pre- 
processed to increase the particle-background contrast. This is achieved combining the POD-based background removal in [24] with the intensity capping [44] and the CLAHE histogram equalization [45]. The PIV interrogation is carried out with the open-source Matlab routine PIVlab [22], using a standard iterative multi-step interrogation (three passes from $64 \times 64$ to $8 \times 8$ ) with spline window deformation and 2D Gaussian sub-pixel interpolation. To increase the flow coherence, limit the random noise and the size of the dataset, the original vector field $\mathbf{u}_{o}=\left(u_{o}, v_{o}\right)$ is low pass filtered and down-sampled with a moving average kernel of size 3 :

$$
\mathbf{u}[i, j, k]=\frac{1}{3} \sum_{p=3 k-2}^{p=3 k} \mathbf{u}_{o}[i, j, p] .
$$

The vector validation is carried out both before and after the down-sampling by setting a threshold on the histogram standard deviation and using the median test [46]. The resulting dataset consists of $n_{t}=3500$ velocity fields having $n_{x} \times n_{y}=63 \times 63$ vectors, with a spatial resolution of about $\Delta x=0.35 \mathrm{~mm}$, and a time resolution of $\Delta t=1 \mathrm{~ms}$. An examplary velocity field is shown in Fig.8.

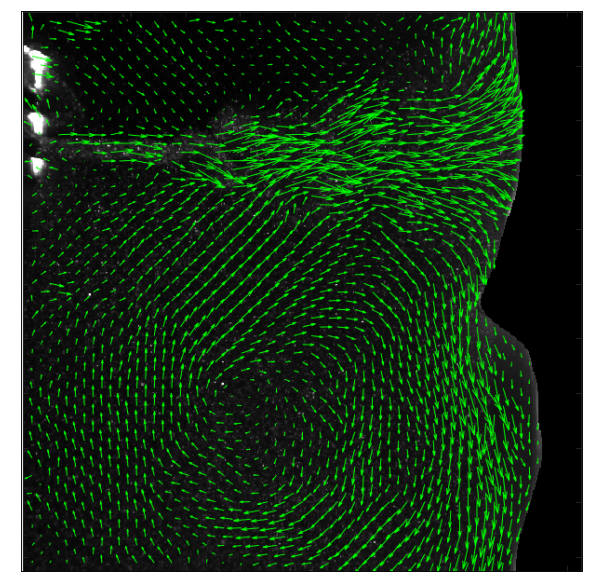

Fig. 8. Down-sampled velocity field with automatic masking of the liquid film interface. The spatial and temporal resolutions are $0.35 \mathrm{~mm}$ and $1 \mathrm{~ms}$ respectively.

The frequency response of the gas flow field is analyzed from the spectral content of the dominant POD modes (see also [25, 26]) of the down-sampled velocity field.

That is, the zero mean shifted data set $\mathbf{u}^{\prime}=\mathbf{u}-$ $1 / n_{t} \sum_{k=1}^{n_{t}} \mathbf{u}_{k}$ is decomposed into a linear combination of modes composed of a spatial structure $\phi_{\mathbf{r}}[i, j]$, a temporal evolution $\psi_{r}[k]$ and an energy content $\sigma_{r}$ :

$$
\mathbf{u}^{\prime}[i, j, k]=\sum_{r=1}^{n_{t}} \sigma_{r} \phi_{r}[i, j] \psi_{r}[k] .
$$

By definition, the spatial structures $\phi_{r}$ are eigenvectors of the spatial correlation $C$ matrix; the temporal evolutions $\psi_{r}$ are eigenvectors of the temporal correlation matrix $K$; the energy contents $\sigma_{r}$ are the square roots of the common eigenvalues of $C$ and $K$. These matrices are defined as

$$
\begin{aligned}
& K[i, j]=\frac{1}{2 n_{s}} \sum_{m=1}^{n_{x}} \sum_{n=1}^{n_{y}} \mathbf{u}^{\prime}[m, n, i] \mathbf{u}^{\prime}[m, n, j] \\
& C[\mathbf{i}, \mathbf{j}]=\frac{1}{n_{t}} \sum_{m=1}^{n_{t}} \mathbf{u}^{\prime}[\mathbf{i}, m] \mathbf{u}^{\prime}[\mathbf{j}, m]
\end{aligned}
$$

where $n_{s}=n_{x} \times n_{y}$ is the number of spatial points, $\mathbf{i}, \mathbf{j}=1, \ldots n_{s}$ are the matrix linear indices. A typical POD spectrum is shown in Fig.9.

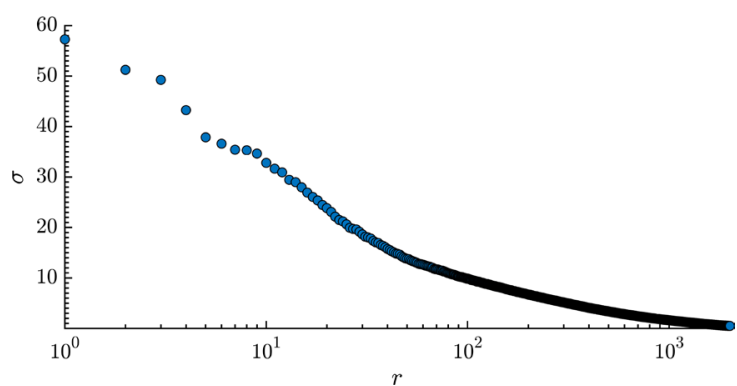

Fig. 9. Energy content in each POD mode for an exemplary test case. Typically, the first five modes capture a large amount of the total energy in the dataset, while following gentle decay is due to the highly turbulent nature of the flow field.

While the number of modes required to capture the large scale features of the flow is of the order of ten, the energy decay in the following modes remains rather smooth, due to the highly turbulent nature of the dataset. In all the experiments performed, however, the dominant modes are characterized by a highly harmonic behavior, which accounts for a large scale and coherent oscillation of the impinging jet flow.

The frequency content of the first six modes for the exemplary test case presented in Fig.8 is shown in Fig.10. The harmonic response of the jet oscillation is present in the dominant mode related to such an instability. This response is linked to the run-back film pulsation, characterized by the spectrum in Fig.7b, as discussed in Section 5. The characteristic spectra of the jet flow, therefore, is taken from the power spectral density of the temporal evolution of the first POD modes, i.e. $\boldsymbol{y}_{J}=\left|\hat{\psi}_{1}\right|(f)$.

\subsection{Final Coat Characterization}

The TR-LAbs measures the liquid film thickness contour map from the amount of light absorbed from a backlighting source. The absorption is increased by means of a Methylene blue colorant, which absorption spectrum match the emission spectrum of the light source. For sufficiently diluted compounds, the light absorption and the film thickness are linked by the Beer-Lambert's law:

$$
h(x, y, t)=\frac{1}{\gamma} \ln \left(\frac{I_{0}(x, y)}{I(x, y, t)}\right)=\frac{1}{\gamma} A(x, y, t),
$$




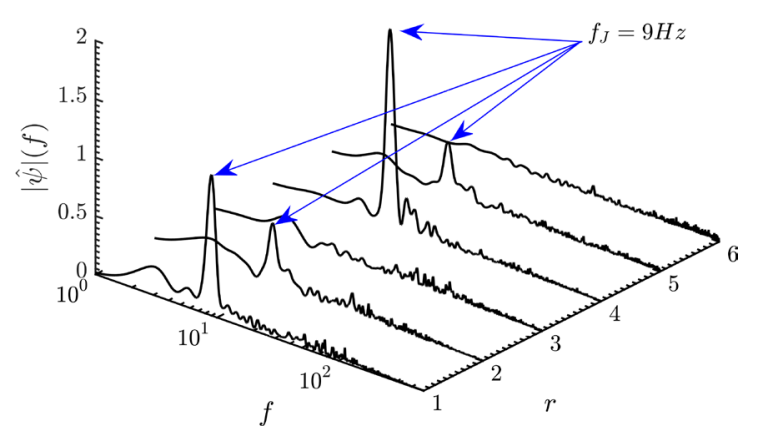

Fig. 10. Exemplary spectra of the first five dominant POD temporal modes $\psi(t)$ of the TR-PIV velocity field of the jet flow. The pulsation of the run back flow (spectra shown in Fig.7b) introduce an harmonic response on the jet flow. Test case: $\hat{Z}=16, U_{J}=29 \mathrm{~m} / \mathrm{s}, U_{s}=0.35 \mathrm{~m} / \mathrm{s}$.

where $I_{0}(x, y)$ is the reference light intensity, taken from the dry test section (see Fig.5a), $I(x, y, t)$ is the intensity of the light transmitted through the liquid layer (cf. Fig.5b) and $\gamma$ is the absorption coefficient, which mostly depends on the colorant concentration. Both light intensities $I_{0}$ and $I$ are measured in terms of image gray levels by the camera. For a colorant concentration of $21 \mathrm{mg} / \mathrm{Kg}$, the absorbance coefficient is $\gamma=0.75 \pm 1 \% \mathrm{~mm}^{-1}$.

The major difficulty encountered in this work is the motion of the cylinder which makes the time independence of the reference image questionable. While this image $I_{0}(x, y)$ is taken as a time average of a video recording with the dry test section, defects in the acrylic glass and the entrainment of air bubbles results in moving regions where the thickness measurement is compromised. An example of thickness reconstruction obtained by using eq.(11) is shown in Fig.11a.

An image processing routine was therefore developed to remove these defects in the post-processing step. The range of acceptable thickness measurements is first set by a threshold in the standard deviation of the thickness map. The outliers regions are converted to a binary image and the connected pixel clusters are labeled using connectivity analysis ([47], Fig.11b). Connected areas smaller than a user defined threshold are removed and re-interpolated by means of the penalized least squares method proposed in [48]. A typical liquid film interface reconstruction, after the outliers removal and the smoothing step, is shown in Fig.11-c.

For the purpose of this work, it was of interest to evaluate the film thickness average, the interface smoothness and a characteristic liquid film spectra. The thickness average is computed by averaging the film reconstruction both in space and in time:

$$
h_{f}=\frac{1}{n_{s} n_{t}} \sum_{\mathbf{i}=1}^{n_{s}} \sum_{k=1}^{n_{t}} h[\mathbf{i}, k]
$$

where $\mathbf{i} \in\left[1, n_{s}\right]$ are spatial points (pixels) and $k=$ $\left[1, n_{t}\right]$ is the image index number within a video. The interface smoothness is evaluated by considering the peak to peak amplitude within a typical interface wave. This is

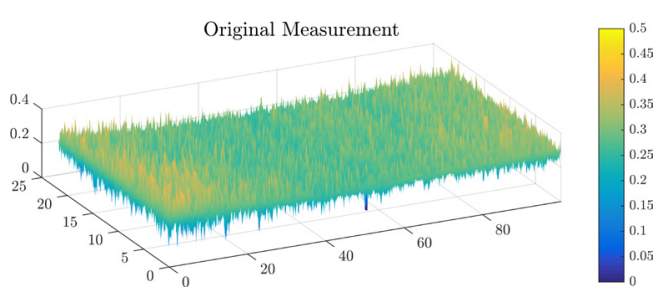

a)

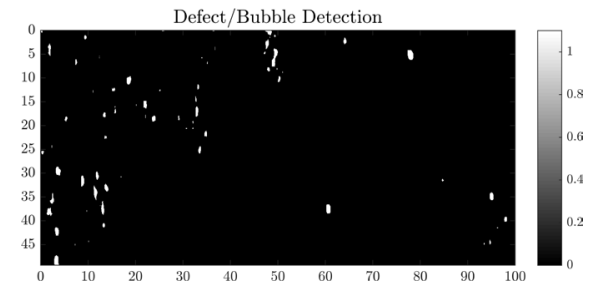

b)

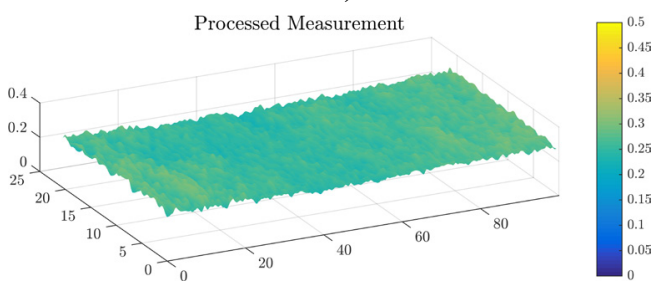

c)

Fig. 11. a) Example of TR-LAbs measurement with no pre-processing; b) bubble/defect detection using morphological labeling and histogram threshold; c) processed measurement with outliers interpolation and smoothing.

evaluated by averaging in space the difference between the envelope of the wave peak $h_{M}[\mathbf{i}]$ and the envelope of the wave minima $h_{m}[\mathbf{i}]$ :

$$
h_{p p}=\frac{1}{n_{s}} \sum_{\mathbf{i}=1}^{n_{s}}\left(h_{M}[\mathbf{i}]-h_{m}[\mathbf{i}]\right)
$$

where the envelopes are defined as:

$$
\begin{aligned}
h_{M}[\mathbf{i}] & =\max _{k}^{n_{t}}\{h[\mathbf{i}, k]\} \\
h_{m}[\mathbf{i}] & =\min _{k}^{n_{t}}\{h[\mathbf{i}, k]\}
\end{aligned}
$$

Finally, the characteristic spectra of the liquid is computed as the spatial and the temporal ensemble average of the stream wise (thus along the image rows) thickness film profiles, that is:

$$
\mathcal{H}_{f}[n]=\frac{1}{n_{t} n_{y}} \sum_{i=1}^{n_{y}} \sum_{k=1}^{n_{t}} H_{i}^{k}[n]
$$

where $H_{i}^{k}[n]$ is thickness spectrum computed along the columns $j \in\left[1, n_{x}\right]$, for each $i \in\left[1, n_{y}\right]$ raw of the $k \in$ $\left[1, n_{t}\right]$ image:

$$
H_{i}^{k}[n]=\frac{2}{n_{x}} \sum_{j=1}^{n_{x}} h[i, j, k] \exp \left(\frac{-2 \pi \mathrm{i}(j-1)(n-1)}{n_{x}}\right) .
$$




\section{Results and Discussion}

Keeping the cylinder velocity of $U_{s}=0.35 \mathrm{~m} / \mathrm{s}$, the experimental campain consists of six jet velocities (from $U_{J}=23 \mathrm{~m} / \mathrm{s}$ to $U_{J}=39 \mathrm{~m} / \mathrm{s}$ ) at two stand-off distances, namely $\hat{Z}=12$ and $\hat{Z}=16$. The analysis is herein divided in terms of statistical quantities (Section 5.1) and frequency analysis (Section 5.2).

\subsection{Averaged Quantities of the Final Coat}

The measured average film thickness is shown in Fig.12, as a function of the dimensionless pressure gradient and compared with the OD wiping model in eq.(2). The pressure gradient for the experimental points is computed from eq.(3) and the measurements are scaled with respect to the optimal thickness $h_{0}=\sqrt{\left(v U_{s} / g\right)}$.

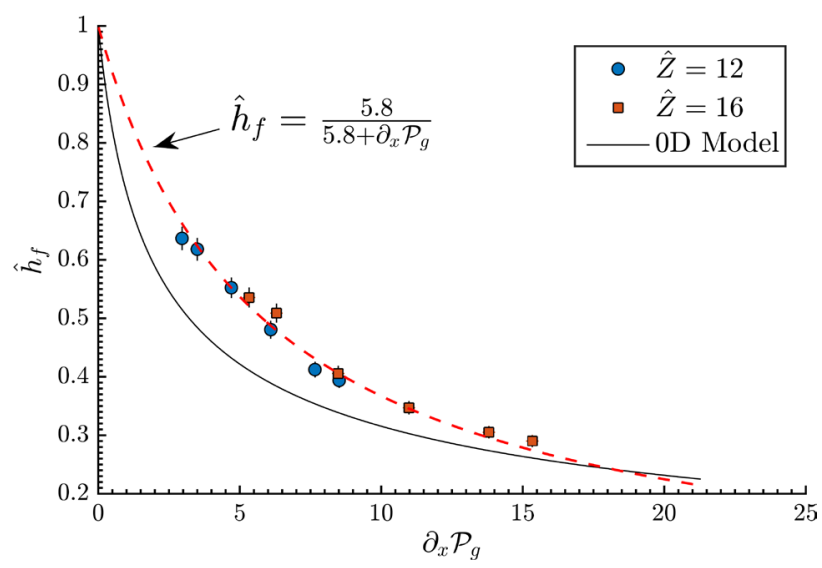

Fig. 12. Comparison of the measured average film thickness of the final coat $\hat{h}_{f}$ in dimensionless form with the OD wiping model in eq.(2), as function of the dimensionless pressure gradient in eq.(3).

As expected, the 0D model overestimates the wiping capabilities at the lowest pressure gradients, while the agreement with the experimental points improves for stronger wiping performances. A simple rational correlation is proposed to fit the experimental points. This correlation, shown in Fig.12 is constructed such that $\hat{h}_{f}=1$ for $\partial_{x} \mathcal{P}_{g} \rightarrow 0$ and can be considered as an empirical correction to the eq.(2) for $\partial_{x} \mathcal{P}_{g}<18$.

The peak to peak amplitudes of the waves in the final coat are shown in Fig.13, scaled with respect to the optimal thickness and correlated to the final coating thickness regardless of the stand off distance or the jet velocity. Such correlation suggests that the characteristic of the final coat solely depends on the coating thickness, and therefore on the jet pressure gradient and shear stress at the impact, regardless of the conditions at which the wiping takes place $\left(\Delta P_{N}, Z, d, U_{s}\right)$. Concerning the order of magnitudes, in the range $5-10 \%$, one should take into account that the film thickness is analyzed at a distance of about $L \approx 1 / 2 \pi R \approx 330 \mathrm{~mm}$, with $R$ the cylinder radius, from the wiping point. In all these cases then, the waves reach the measurement test section $\approx 1 s$ after the wiping has taken place and during which they are subject to the damping action of surface tension. Concerning the trend, the monotonically increasing behavior implies that stronger wiping conditions $\left(h_{f} \rightarrow 0\right)$ lead to smaller waves in absolute values. As the proportionality of the amplitude is $\hat{A_{f}} \propto \hat{h}_{f}^{0.6}$ these results suggest that in relative terms the amplitude of the coating non-uniformities increases $\left(\hat{A_{f}} / \hat{h}_{f} \propto \hat{h}_{f}^{-0.4}\right)$ for stronger wiping.

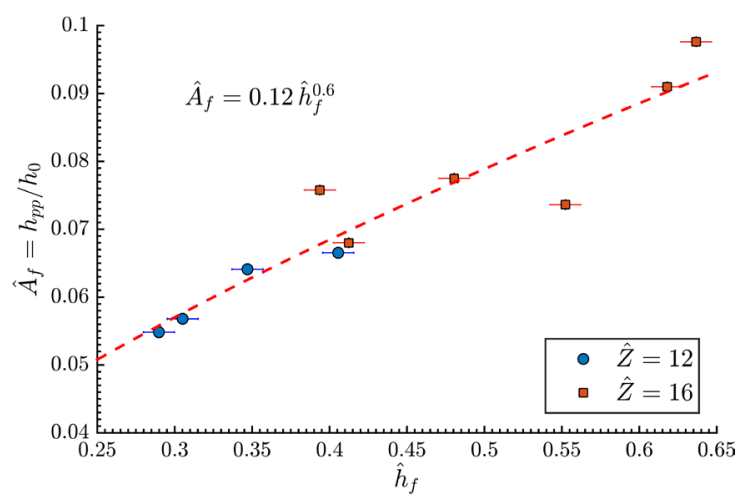

Fig. 13. Peak to peak amplitude of the waves in the final coat.

Whether such trends can be justified on the basis of a liquid film instability requires further investigation, although several authors have shown, using standard linear stability analysis, that the flow in the final coat is neutrally stable $[49,50]$. On the other hand, on the basis of the average thickness curve in Fig.12, such trend is in line with the hypothesis that undulation in the final coat results from an instability of the impinging jet flow, which produces time variations of the pressure gradient [15-18]. The wiping curve in Fig. 12 predicts, in fact, a saturation of the final thickness at the largest pressure gradients, which implies that thinner coats are less sensitive to variations in the wiping conditions than thicker ones.

\subsection{Frequency Analysis of the Wiping Process}

The characteristic spectra for the run back liquid film $\left(\mathcal{H}_{R}\right)$, the gas jet flow $\left(\mathcal{Y}_{J}\right)$ and the final coating thickness $\left(\mathcal{H}_{f}\right)$, computed as described in Section 4, are shown for the six gas jet velocities in Fig. 14 for the stand-off distance $\hat{Z}=12$ (left) and $\hat{Z}=16$ (right). Each spectrum is divided by its maximum for plotting purposes.

Interestingly, the dominant peak in spectra of the gas jet always match the one in the spectra of the run back liquid flow and the corresponding frequency increases monotonically with the velocity of the impinging jet. To investigate the role of the jet flow and that of the liquid film in this spectral matching, these results are scaled using a different characteristic time for the two flows in Fig.15. For the liquid film, the characteristic time considered is based on the liquid viscosity and the withdrawal speed $[t]=h_{0} / U_{s}=\sqrt{v /\left(U_{s} g\right)}$ and the resulting dimensionless frequency is shown in Fig.15a) as a function of the final coating thickness $\hat{h}_{f}$ and the run back film thickness $\hat{h}_{R}$. The latter is computed by introducing the measured 

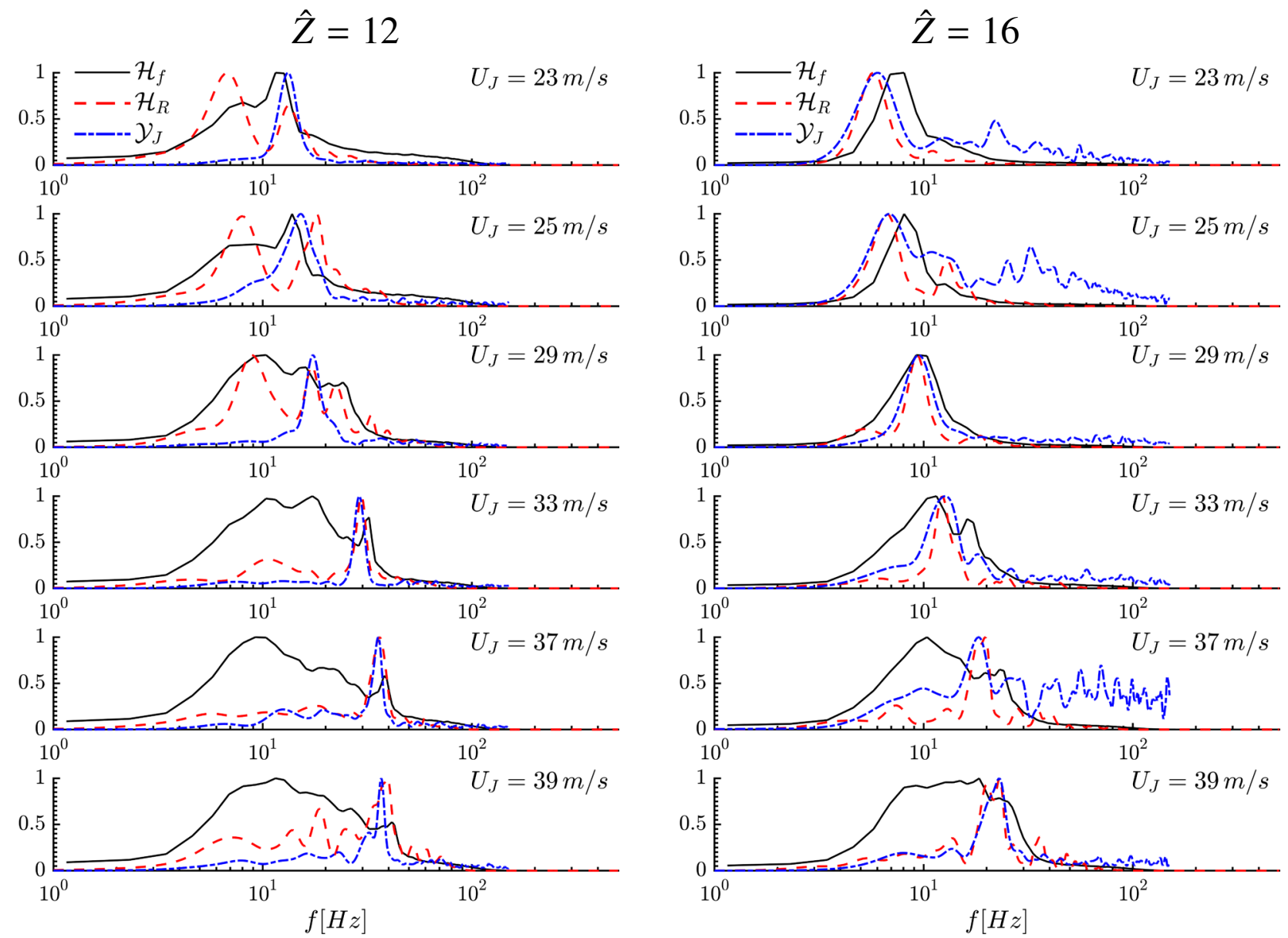

Fig. 14. Spectra of the final coating layer $\left(\mathcal{H}_{f}\right.$, black continuous lines), the run back liquid flow $\left(\mathcal{H}_{R}\right.$, red dashed lines) and the gas jet flow $\left(\mathcal{Y}_{J}\right.$, blue dotted lines) for $\hat{Z}=12$ (left) and $\hat{Z}=16$ (right) for six velocities of the impinging jet flow. These spectra are normalized with respect to their peak; the amplitude of the coating defect is discussed in Fig.13.

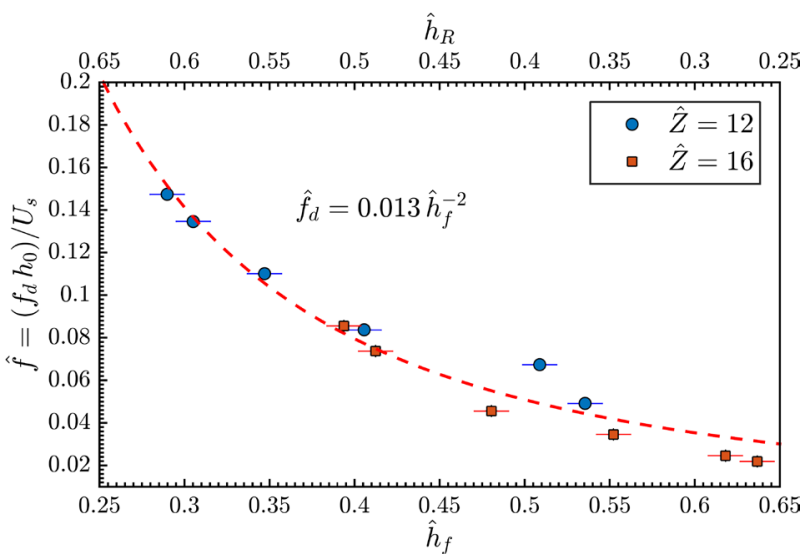

a)

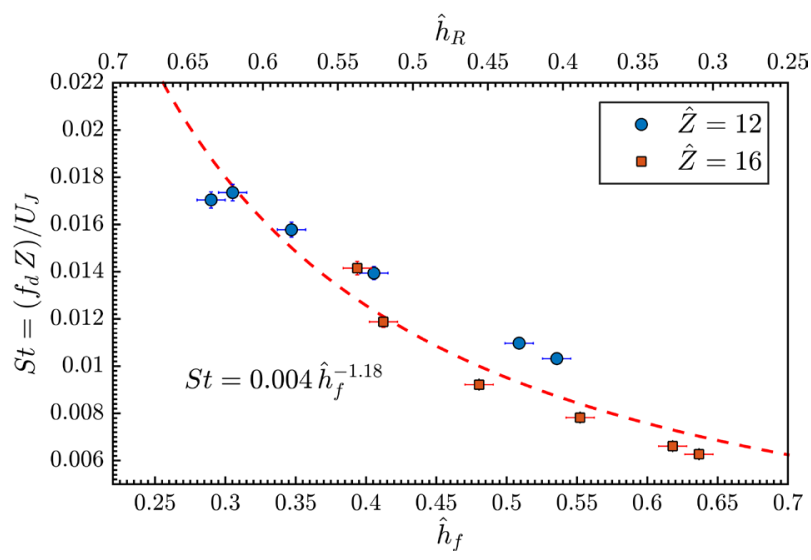

b)

Fig. 15. Dimensionless correlation for the dominant frequency in the run back liquid film and in the impinging jet flow scaled with respect to the liquid film parameters $\left(\hat{f}=f h_{0} / U_{s}\right.$, Fig. a) and with respect to the gas jet parameters $\left(S t=f Z / U_{J}\right.$, Fig. b). 


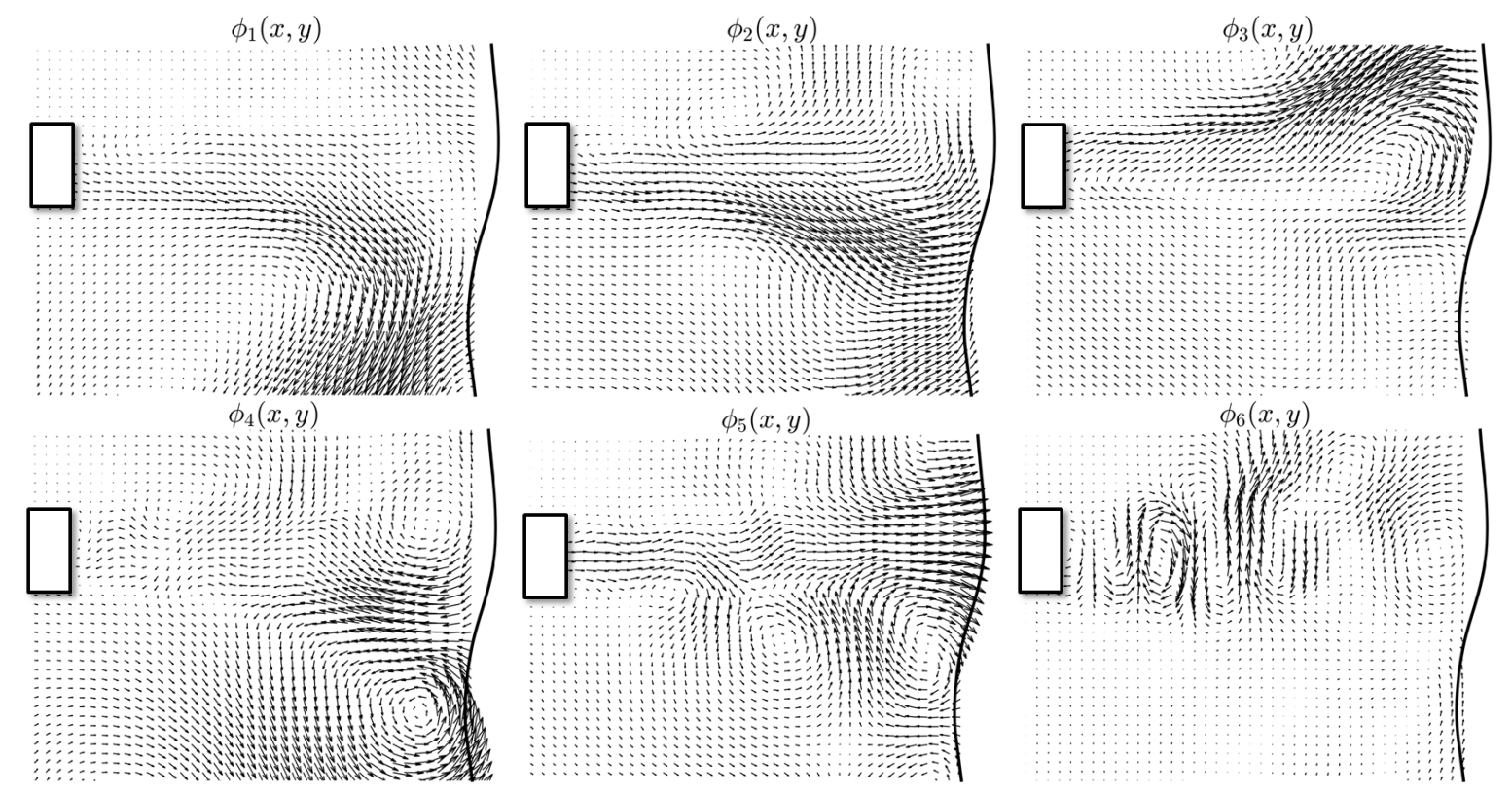

Fig. 16. Spatial structures $\phi[i, j]$ of the six POD modes of the TR-PIV dataset, associated with the spectra shown in Fig.10. The combination of the first five modes describes a sustained oscillation of the impinging jet, linked to the run back flow pulsation. Modes above the 5th are linked to shear layer instabilities and characterized by much higher frequencies, disregarded in this work.

average thickness in the final coat in the universal cubic equation in Fig. 2 for the far field conditions.

The collapsing of the measurements with the final thickness is once again showing that the interaction between the two flows is solely governed by the wiping strength, regardless of other jet parameters. In particular, the frequency of the run back flow pulsation increases for stronger wiping, and thus increases with the run back flow thickness. This result is expected, both on a qualitative level and in terms of order of magnitude, for the most amplified waves in the linear stability analysis of a liquid film falling freely over a vertical fixed wall [36]. A quantitative agreement is on the other hand not expected since the run-back flow is in a highly nonlinear regime and strongly sheared by the impinging gas jet. It is therefore reasonable to argue that the run back flow pulsation is the result of a liquid film instability, that would exist even for an ideally stable impinging gas jet.

Concerning the gas jet oscillation, the dominant frequency is scaled with respect to the jet flow parameters, i.e. taking a reference time scale as $[t]=Z / U_{J}$, in Fig.15b. The dimensionless frequency of the jet oscillation is about one order of magnitude lower than what is observed for a gas jet impinging on a solid interface which models the shape of the run back flow interface, for which it is found in $[18,23,51]$ a constant Strouhal number $S t=f Z / U_{J} \approx 0.07$. Moreover, while the Strouhal number of the oscillation for a gas jet impinging on these solid models is independent of the stand-off distance and the maximum interface height $[18,23,51]$, the Strouhal number in the wiping condition is here found to vary with the stand off distance and to be solely a function of the wiping performance. This behavior is unexpected for a gas jet impinging on a solid wall, and it is therefore argued that the jet oscillations are driven by the pulsations of the run back flow.

The hydrodynamic mechanism which is responsible for the link between the run-back flow pulsations and the impinging gas jet oscillation is well captured by the first five POD modes of the TR-PIV data of the jet flow, an exemplary set of which is shown in Fig.16. These modes have in fact a similar temporal evolution, dominated by the frequency of the run-back flow pulsation (in this example, shown in Fig.7b) and visible from their spectra, shown in Fig.10. The combination of these modes describes a sustained oscillation of the impinging jet flow (mostly captured by the modes 1-2-3), driven by the unsteady flow recirculation produced in the run back flow (captured by the modes 1-4-5). Higher modes appear to be uncorrelated (typically starting from the mode 6) with the run back flow pulsation and are linked to shear layer instabilities of the jet flow, occurring at much higher frequencies.

The mechanics of the low-frequency oscillation of an impinging gas jet, driven by the unsteadiness of the impinged interface were reported in a similar configuration in [16-18]. Regardless of the geometrical configuration, several common features can be identified: the periodic deflection of one of the wall jets due to the interface pulsation and the formation of unsteady recirculation produced by the entrainment flow, the impinging jet, and the deflected wall jet.

Finally, while it is clear the pulsation in the run back flow drives the oscillation of the jet, it not clear whether the undulation in the final coat results from the stream-wise propagation of the run back flow pulsation or the oscillations of the impinging gas jet. A perfect match of the peaks 
of $\mathcal{Y}_{J}$ and $\mathcal{H}_{f}$ appears at the lowest velocities for both the stand-off distances, but vanishes at the largest velocities, for which the frequency spectra in the final coat broadens considerably. Particularly interesting are the cases at low velocity and close stand-off distance, where the run back flow features two harmonic responses, both observed also in the final liquid film. It is therefore reasonable to conclude that the oscillation of the impinging gas jet, produced by the coupled instability between the gas jet flow and the run back liquid film, is one of the key sources for the final coating non-uniformity. However, the discrepancy in the frequency spectra at the fastest jet speed implies that this is not the only source and that the final coat could in principle assume a wavy pattern regardless of the impinging jet flow stability.

\section{Conclusions}

An experimental characterization of a jet wiping process, in which a 2D gas jet impinges on a liquid film to control the coating thickness over a moving substrate, has been presented. After discussing the relevant dimensionless numbers and the scaling laws of this two-phase flow configuration, the jet wiping facility Ondule, developed at the von Karman Institute, was presented together with the measurement test sections used for the temporal and spatially resolved investigation.

These include Time Resolved Particle Image Velocimetry (TR-PIV) and liquid interface tracking via image processing to study the wiping process in the impact region, and 3D Time-Resolved Light Absorption (LAbs) to characterize the interface dynamics of the coating film flow after the wiping.

The statistical properties of the final liquid film confirm the prediction of simplified engineering models of the wiping process, while the frequency analysis of the interface dynamics at the impact, combined with the modal analysis of the jet flow via Proper Orthogonal Decomposition (POD), revealed an unstable and periodic mechanism. In particular, it is shown that over the entire range of experimental conditions, the liquid film wiped from the plate and running back to the bath is unstable and develops a periodic train of large amplitude waves. These waves unsteadily confine the impinging gas jet, periodically producing a large scale recirculation which destabilizes the gas flow and promotes its oscillation. Simple correlations for the characteristic frequency of this coupling phenomenon are given in terms of liquid film and gas jet characteristic scales as a function of the wiping condition, to provide a solid experimental support for validating numerical or theoretical approaches.

As this unsteady phenomenon results in time dependent wiping capabilities of the jet, it is argued that its occurrence can be printed in the final coat and therefore be responsible for the limited coating quality achieved by jet wiped products. However, since the spectral matching between jet oscillations and the waves in the final coat is lost in some of the presented test cases it is concluded that other source of instability of the jet wiping process must exist.

\section{Acknowledgments}

The authors gratefully acknowledge the Belgian FNRS for supporting M. A. Mendez with a FRIA grant, and ArcelorMittal, for the financial support and for the permission to publish the material presented.

\section{List Of Symbols}

\section{Acronyms}

TR-PIV Time Resolved Particle Image Velocimetry

TR-LAbs Time Resolved Light absorption

VKI von Karman Institute

POD Proper Orthogonal Decomposition

PSD Power Spectral Density

CLAHE Contrast-Limited Adaptive

Histogram Equalization

\section{Notation}

[x] Characteristic scale for $x$

$\hat{x} \quad$ Nondimensionalized $\mathrm{x}$

$x_{M} \quad$ Maximum component of $x$

$x_{m} \quad$ Minimum component of $x$

$x_{f} \quad x$ property of the final coat

$x_{R} \quad x$ property of the run-back flow

$x_{s} \quad x$ property of the solid substrate

$x_{j} \quad x$ property of the jet flow

$x_{l} \quad x$ liquid property

$x_{g} \quad x$ gas property

$x_{0} \quad x$ reference quantity

$\mathbf{x}^{\prime} \quad$ mean subtracted quantity

$h_{p p} \quad$ peak to peak amplitude

$u \quad$ velocity vector field

$\mathcal{H}$ characteristic spectrum

$C$ spatial correlation matrix

$K \quad$ temporal correlation matrix

$\phi_{r} \quad$ modes spatial structure

$\psi_{r} \quad$ modes temporal evolution

$\sigma_{r} \quad$ modes energy content

$n_{t} \quad$ number of samples

$n_{s} \quad$ number of spatial points

$n_{x} \quad$ number of columns

$n_{y} \quad$ number of rows

\section{Dimensionless Quantitites}

$R e_{L} \quad$ Reynolds number of the liquid film

$R e_{j} \quad$ Reynolds number of the gas jet flow

$S t \quad$ Strouhal number of the gas jet flow

$y_{J} \quad$ characteristic spectrum of the gas jet flow

$C_{\tau} \quad$ empirical coefficient

$\hat{A}_{f} \quad$ dimensionless peak to peak amplitude

$\zeta \quad$ wiping thickness aspect ratio

$\partial_{x} \mathcal{P}_{g} \quad$ pressure gradient 


\section{Dimensional Quantities}

$\begin{array}{lll}U & \text { velocity } & \mathrm{m} / \mathrm{s} \\ \Delta P_{n} & \text { nozzle stagnation pressure } & \mathrm{Pa} \\ P & \text { pressure } & \mathrm{Pa} \\ Z & \text { nozzle stand-off distance } & \mathrm{mm} \\ d & \text { nozzle opening } & \mathrm{mm} \\ \rho & \text { density } & \mathrm{kg} / \mathrm{m}^{3} \\ \mu & \text { dynamic viscosity } & \mathrm{mPa} . \mathrm{s} \\ v & \text { kinematic viscosity } & \mathrm{m}^{2} / \mathrm{s} \\ \sigma & \text { surface tension } & \mathrm{N} / \mathrm{mm} \\ h & \text { thickness } & \mathrm{mm} \\ h_{0} & \text { optimal thickness } & \mathrm{mm} \\ g & \text { gravitational acceleration } & \mathrm{m} / \mathrm{s}^{2} \\ q & \text { final coat flow rate } & \mathrm{m}^{3} / \mathrm{s} \\ T_{g} & \text { shear stress } & \mathrm{Pa} \\ t & \text { time } & \mathrm{s} \\ R & \text { Cylinder radius } & \mathrm{mm} \\ b & \text { x position where } P=P_{M} / 2 & \mathrm{~mm} \\ \Delta x & \text { spatial resolution } & \mathrm{mm} \\ \Delta t & \text { temporal resolution } & \mathrm{ms} \\ f & \text { frequency } & \mathrm{Hz} \\ \gamma & \text { light absorption coefficient } & \mathrm{mm} \\ I & \text { light intensity } & \mathrm{grayscale}\end{array}$

\section{References}

1. G.E. Cohen, E., Modern Coating and Drying Technology (New York: VCH, 1992)

2. TAPPI, Tech. rep., Atlanta: TAPPI Press (1981)

3. Deryagin, B.M, Levi, S.,M., Film Coating Theory (New York: Focal Press, 1964)

4. Butler, JJ., Beam, D.J, Hawkins, J.C., Iron and Steel Engineer 8, 77 (1970)

5. Thornton, J. A., Graff, H.F., Metallurgical Transactions B 7, 607 (1976)

6. Ellen, C H, Tu, C V, J. Fluids Eng. 106, 399 (1984)

7. Tuck, E.O., Phys.Fluids 9, pp. 2352 (Sept,1983)

8. Buchlin, J.-M., Modelling of Gas Jet Wiping, in Thin Liquid Films and Coating Processes, VKI Lecture Series (von Karman Institute for Fluid Dynamics, 1640 Rhode-Saint-Genese, Belgium, 1997)

9. Elsaadawy, E.A., Hanumanth, G.S., Balthazaar, A.K.S., McDermid, J.R., HryMak, A.N., Forbes, J.F., Metallurgical and Materials Transactions B 38, pp. 413 (2007)

10. Bertin, M.C., Brown, L., Whitehead, R.L., Yrisarri, E., IFAC Proceedings Volumes 11, pp 199 (1978)

11. Fenot, C. Rolland, F., Vigneron, G., Landau, E.D., IFAC Proceedings Volumes 25, pp. 347 (1992)

12. Guelton, N., Lopes, C., Sordini, H., Metallurgical and Materials Transactions B 47, pp 2666 (2016)

13. Myrillas, K., Gosset, A., Rambaud, P., Buchlin, J.-M, Eur. Phys. J. Spec. Top 166, pp. 93 (2009)

14. Myrillas, K., Rambaud, P., Mataigne, J.-M., Gardin, P., Vincent, S., Buchlin, J.-M, Chem. Eng. Process. 68, pp. 26 (2013)

15. Pfeiler, C., Ebi, W., Reiss, G. Riener, C.K., Angeli. G. Kharicha, A., Steel Res. Int. 87, pp 1 (2017)
16. Mendez, M.A., Dynamics of Gas Jet Impingement on Vertical Falling Liquid Films, in Proceedings of the 6th Symposium of VKI PhD Research (Rhode Saint Genese, BE, 2015)

17. Mendez, M.A., Buchlin, J.-M., A research methodology to study jet wiping processes, in Proceedings of the 11th European Coating Symposium ECS2015 (Eindhoven, NL, 2015)

18. Mendez, M.A. , Oscillation of a Planar Gas Jet Impinging on a Deformable Wall, in Proceedings of the 7th Symposium of VKI PhD Research (Rhode Saint Genese, BE, 2016)

19. Lacanette, D, Gosset, A., Vincent S., Buchlin, J-M, Arquis E., Phys. Fluids 18, 042103 (2006)

20. Gosset, A., Buchlin, J.-M., Journal of Fluids Engineering 129, 466 (2007)

21. Dubois, M., Reithmuller, M.L., Buchlin, J.-M., Arnalsteen, M., The Gas-Jet Wiping Limit: The Splashing Phenomenon, in GalvatechConference (Chicago, Iron and SteelInstitute, 1995), pp. 667-673

22. Thielicke, W., Stamhuis, E.J., Journal of Open Research Software 2, 30 (2014)

23. Mendez, M.A., Buchlin, J.-M., Quantitative flow visualization of confinement-driven instabilities of an impinging slot jet, in 11th International Symposium on Particle Image Velocimetry (PIV2015) (Santa Barbara, California, US, 2015)

24. Mendez, M.A., Raiola, M., Masullo, A., Discetti,S., Ianiro,A., Theunissen, R., Buchlin, J.-M., Exp Therm Fluid Sci 80, 181 (2017)

25. P. Holmes, J. Lumley, G. Berkooz, Cambridge University Press (1996)

26. L. Sirovich, Quart. Appl. Math. 45, 561 (1987)

27. Gosset, A., Measurement techniques for unstable film flows, in VKI Lecture Series on Thermo-hydraulic Instabilities (2006), pp. 1-25

28. Ozgen, S., Carbonaro, M, Sarma, G.S.R., Phys. Fluids 14, pp. 3391 (2002)

29. Mendez, M.A., Scheid, B, Buchlin, J-M, Chemical Engineering Science 170, 122 (2017), 13th International Conference on Gas-Liquid and Gas-LiquidSolid Reactor Engineering

30. Yoneda H., Ph.D. thesis, University of Minnesota, Minneapolis, MN (1993)

31. Beltaos,S.,Rajaratnam,N., J. Hydraul. Res. 11, 29 (1973)

32. Tu, C.V., Wood, D.H., Exp Therm Fluid Sci 13, 364 (1996)

33. Saydlowski, B. K., Air knife (1966)

34. Brezinski, J.P., Method and apparatus for coating paper (1965)

35. Bicknell, J.A., Method and apparatus for coating paper (1964)

36. Kalliadasis, S., Ruyer-Quil, C. , Scheid, B., Velarde, M.G., Falling Liquid Films, Vol. 176 (Springer, Applied Mathematical Sciences, 2012)

37. Groenveld, P., Chemical Engineering Science 25, 1267 (1970) 
38. De Ryck, A., Quéré, D., J. Colloid Interface Sci. 203, pp. 278 (1998)

39. Spiers, R.P., Subbaraman, C.V., Wilkinson, W. L., Chem. Eng. Sci. 29, pp. 389 (1974)

40. Mendez, M.A., Nemeth, L., Buchlin, J.-M, EPJ Web of Conferences 114, pp. 1 (2016)

41. Savitzky, A., Golay, M. J. E., Anal. Chem. 36, 1627 (1964)

42. Welch, P. D., IEEE Transactions on Audio and Electroacoustics AU-15, 70 (1967)

43. Oppenheim, A. V.; Schafer, R. W., Digital signal processing (Prentice-Hall, 1975)

44. Shavit, U., Lowe, R., Steinbuck, J., Exp. Fluids 42, 225 (2007)

45. Pizer, S.M., Amburn, E.P., Austin, J.D., Cromartie, R., Geselowitz, A., Greer, T. Romeny, B.T.H., Zim- merman, J.B., Computer vision, Graphics, and Image Processing 39, 355 (1987)

46. Westerweel, J., Scarano, F., Exp. Fluids 39, 1096 (2005)

47. Gonzalez, R. C., Woods, R. E., Digital Image Processing (Prentice-Hall, Inc., 2014)

48. Garcia, D., Computational Statistics and Data Analysis 54, 1167 (2010)

49. Tu, CV, Ellen, C H, Stability of Liquid Coating in the Jet Stripping Process, in 9th Australasian Fluid Mechanics Conference (1986)

50. Hocking, G. C., Sweatman, W. L., Fitt, A. D., Breward, C., Journal of Engineering Mathematics 70, 297 (2011)

51. Mendez, M.A., Scelzo, M.T., Buchlin, J.-M., Exp Therm Fluid Sci 91, 256 (2018) 